\title{
Optimal Measurement Model for the Assessment of Cell Adhesive Force by Using the Dielectrophoresis Force
}

\author{
Jeng-Liang Lin ${ }^{\mathrm{a}}$, Chyung Ay ${ }^{\mathrm{b}}$, Jie-Yu Cheng ${ }^{\mathrm{c}}$, Chao-Wang Young ${ }^{\mathrm{d}}$ \\ ${ }^{a, b, c, d}$ Department of Biomechatronic Engineering, National Chiayi University, No.300 University Rd., Chiayi, \\ 60004, Taiwan
}

\begin{abstract}
The objective for this research is to assess the optimal measurement model for cell adhesion force. The Human Umbilical Vein Endothelial Cell Line (ECV304) was cultured on a type of biomedical material, polydimethylsiloxane (PDMS). The research also studied the parameters such as alternatives of working solution, styles of PDMS substrate, driving frequency and collagen smearing etc. The result showed the cells cultured on the large area substrate with $2 \mathrm{~mm}$ structural spacing and the small area substrate with $100 \mu \mathrm{m}$ structural spacing have better adhesive force. It was also clear to find that large area substrates also showed faster cell growth and expansion. They are more suitable as culture substrates for the measurement of cell adhesion force. As for work solution, $2 \%$ glucose solution that has relative low conductivity and concentration has the best measurement that effectively obtained cell adhesion force.
\end{abstract}

Index Terms: Polydimethylsiloxane; Endothelial Cell; Dielectrophoresis Force; Cell Adhesive Force

(C) 2012 Published by MECS Publisher. Selection and/or peer review under responsibility of the International Conference on E-Business System and Education Technology

\section{Introduction}

Many studies show that cells must attach and adhere to a substrate before they can start to spread on the substrate, proliferate, differentiate, and migrate. Therefore, the adhesion between cells and cells, or between cells and substrates, is an important aspect of cell-substrate behaviors.

Several studies have investigated the cell adhesion strength from mechanical point of view. In the past researches, cell adhesion strength has been studied as centrifugation force by centrifuge, tensile force by micropipette manipulation, shear force by parallel flow chamber and chemical binding force by atomic force microscope[1][2][3][4]. However, centrifugation force by centrifuge and shear force by parallel flow chamber can not work on a single cell and hence the measured strength of cell adhesion may not be accurate. Tensile force by micropipette manipulation and chemical binding force by atomic force microscope are both complicate and expensive techniques for the analysis of cell adhesion strength. DEP is the phenomenon in which a particle, such as a living cell, is polarized and moved by the electrical gravity in a non-uniform electric

E-mail address: ${ }^{\text {jillin@ @ail.ncyu.edu.tw; }}{ }^{\text {bcay@ mail.ncyu.edu.tw; }}$ 's094218@mail.ncyu.edu.tw; ${ }^{\text {d }}$ youngcw@ mail.ncyu.edu.tw 
field [5]. Like gel electrophoresis, DEP can move neutral particles in a non-uniform AC electric field, for the separation and analysis of a variety of biological particles such as cells, DNA, and viruses[6][7][8]. In here, we utilized the DEP force acting on the cells to induce spatial movement for studying the cell adhesion strength. DEP may provide a new and cheap technique in cell adhesion measurement.

This study uses DEP to investigate adhesion force between cells and substrata. The experiment procedure in this study cultures cells on the substrate surface and then moves them under a non-uniform electric field. The cell adhesion force was calculated when the cell was moved away from the seeding position.

\section{Materials and Mathods}

\subsection{Dielectrophoresis Theory}

When an electrically neutral cell is placed in an electrical field, the cell can be polarized by the external force of the electrical field. DEP force is a phenomenon in which a force is exerted on a dielectric cell when it is subjected to a non-uniform electric field. The most commonly used electrode geometry to generate the nonuniform electric field. Cells will then move towards stronger or weaker regions of the electrical field. The movement of the cells depends on the cells properties, working solution, and the strength of the electrical field.

The dielectric constant $\varepsilon$ (farads $/ \mathrm{m}$ ) for the suspension solution of a living cell indicates the tendency of cell polarization when partial charges of the cell are subject to an electric field. Conductivity $\sigma(\mathrm{S} / \mathrm{m})$ can indicate the tendency for the free charges on the cell to move in an electric field, and usually depends on the solution ion content, dissociated ion concentration, charge amount and ion mobility. In this study, bioparticles are represented as cells. The average dielectrophoretic force acting on a cell, which immersed in a medium and exposed to a spatially non-uniform can be described by [9]:

$$
\mathrm{F}_{\mathrm{DEP}}=2 \pi \varepsilon_{\mathrm{m}}\left[\frac{\varepsilon_{\mathrm{p}}-\varepsilon_{\mathrm{m}}}{\varepsilon_{\mathrm{p}}+2 \varepsilon_{\mathrm{m}}}+\frac{3\left(\varepsilon_{\mathrm{m}} \sigma_{\mathrm{p}}-\varepsilon_{\mathrm{p}} \sigma_{\mathrm{m}}\right)}{\tau\left(\sigma_{\mathrm{p}}+\sigma_{\mathrm{m}}\right)^{2}\left(1+\omega^{2} \tau^{2}\right)}\right] \mathrm{r}^{3} \nabla \mathrm{E}^{2}
$$

Where in this study some constants be assumd:

$\varepsilon_{0}:$ dielectric constant in vacuum, $8.854 \times 10^{-12}$ (farads $\left./ \mathrm{m}\right)($ Ogata et al., 2001)

$\varepsilon_{\mathrm{m}}$ : dielectric constant of solution (glucose), $76.5 \varepsilon_{0}$ (farads/m) (Mckee et al., 2000)

$\varepsilon_{\mathrm{p}}$ : dielectric constant of biological particle, $60 \varepsilon_{0}$ (farads $\left./ \mathrm{m}\right)$ (Morgan and Green, 2003), $3.53(\mu \mathrm{S} / \mathrm{cm})$

$\sigma_{\mathrm{m}}$ : Electric conduction coefficient of solution (glucose)

$\sigma_{\mathrm{p}}:$ Electric conduction coefficient of biological micro-particle, 0.5 (S/m) (Morgan and Green, 2003)

$\tau:$ characteristic time of dipole charge(sec)

$\mathrm{r}$ : cell radius $(\mathrm{m})$

$\nabla \mathrm{E}^{2}:$ gradient of electric field squared $(\mathrm{V} / \mathrm{m})^{2}$

$\omega$ : electric field frequency $(\mathrm{rad} / \mathrm{sec})$

Hence, $\mathrm{DEP}$ force $\left(\mathrm{F}_{\mathrm{DEP}}\right)$ is can be calculated from the above equation. Since the DEP force is directed along the gradient of electric field intensity, $\nabla \mathrm{E}^{2}$, and electric field intensity $(\mathrm{E})$ is proportional to the electric potential $(\mathrm{V})$ which works in the electric field when the length of medium between two electrodes is constant. 


\subsection{Materials}

The human endothelial cells, ECV304 was obtained from America Type Culture Collection (ATCC). SYLGARD ${ }^{\circledR} 184$ silicone elastomer kit was purchased from Dow Corning (Taipei, Taiwan). All culture materials were purchased from Gibco (Grand Island, NY, USA) and all chemicals of reagent grade were obtained from Sigma (St Louis, MO).

\subsection{The measurement of cell adhesion force}

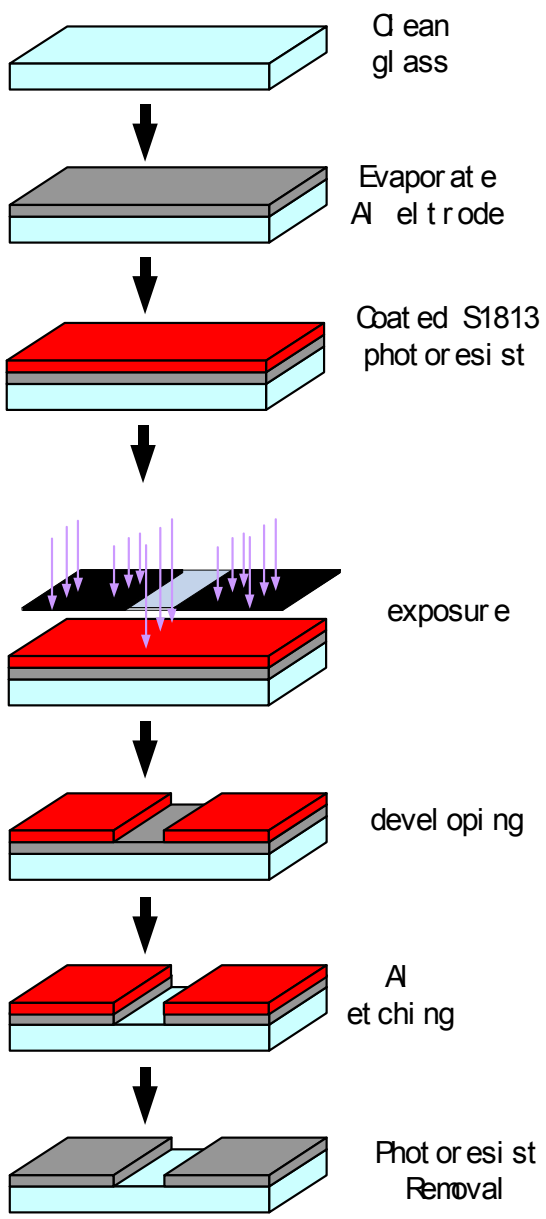

Fig. 1. (a)

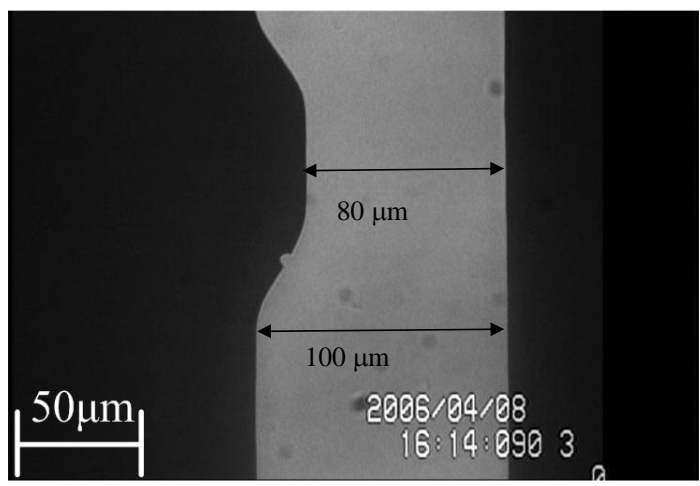

Fig. 1. (b)

Fig. 1. Micro-electrode process and product

The experiment utilized micro-processing technology to etch a glass plate with two different width electrodes to produce non-uniform electric field (Fig. 1a). For DEP force generation, electrical potential was supplied to the aluminum electrode (Fig. 1b) by a function generator and a power amplifier to produce an 
uneven electrical field. The scheme of dielectrophoresis (DEP) force measurement system is shown in Fig. 2. For measurement of cell adhesion strength, cells were first seeded on collagen type 1 and fibronectin coated PDMS membranes respectively. At certain time intervals after cell seeding, the PDMS membranes were covered with two different width electrodes plate (deposition aluminum with a height of $800 \mathrm{~nm}$; the shorted distance between electrodes set at $80 \mu \mathrm{m}$, with the greatest distance between electrodes at $100 \mu \mathrm{m})$ to produce DEP force with the supply of electrical charge (Fig. 1b). During the measurement of cell adhesion strength, electrical potential was increased in the rate of one-third voltage per second from 0 to $10 \mathrm{~V}$ and then changed to one-tenth voltage per second until the focal cell was detached from the PDMS surface. Till cell detached from the PDMS surface, the electrical potential (V) is recorded to be the strength of cell adhesion.

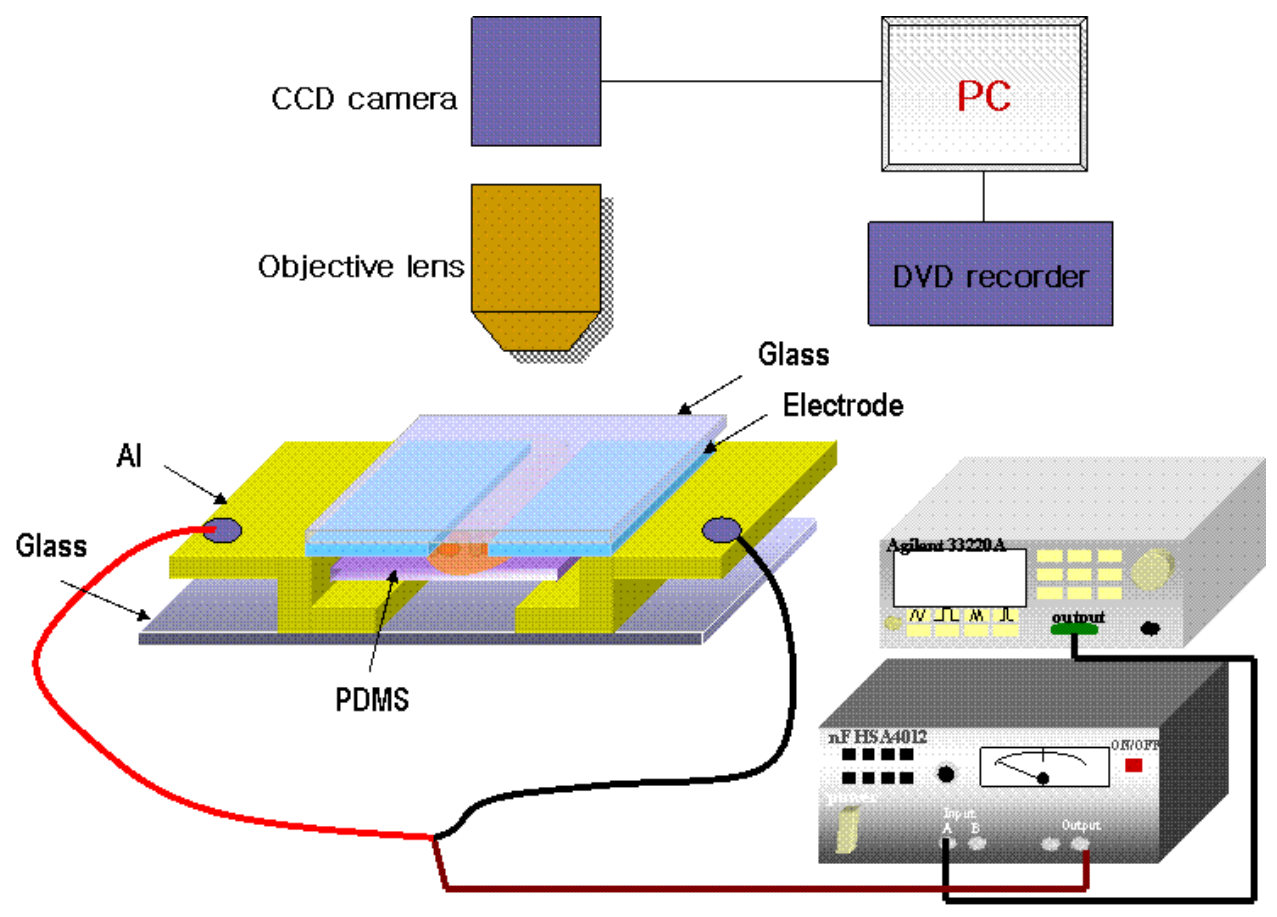

Fig. 2. System Diagram

\subsection{Methodology}

The object of this study is to find out the optimal measurement cell adhesive force, which works in the optimal environment parameter, such as solution, working frequency, collagen smearing etc., to avoid the bubble formation in the solution. It is to use collagen smearing to culture endothelial cells on different PDMS micro-structure substrate, investigate the experimental factors to affect cell adhesion, optimal smearing method, possible issues and solutions to obtain the desired cell growth condition.

Since the cell culture substrate in the research was polydimethylsiloxane (PDMS) which hydrophobicity prevented collagen smearing, microstructure was built on PDMS to hold the solution in the cell culture area by capillary effect. Investigation was also made between structured and non-structured PDMS and for different structural spacing. Through this, whether structure could affect adhesion significantly would be known, as well as whether different structural spacing when cells have different growth space would generate different physiological mechanism. 


\subsubsection{Optimal measurement experiments}

\subsubsection{Selection of Work Solution}

Dielectric property, conductivity and dissociation degree for different work solutions would change the effect of dielectrophoresis force on cell behavior. Overly high osmolarity can cause leakage of water inside cell membrane and crenation; overly low osmolarity can cause water from work solution to enter cell membrane and expand, or even rupture, the cell. Thus, selection of solution and concentration is an essential parameter in the experiment.

\subsubsection{Style of cell culture substrate}

The research used collagen smear on different PDMS micro-structure substrates for cell adhesion. Since PDMS is hydrophobic, when collagen is smeared on PDMS, its contact angle with PDMS is large due to surface tension and beads are formed. So it is necessary to have an integrated smearing process to provide each cell with identical growth condition. In this study, there are two ways to find out which style is better to cell culture. One way is compared no-modified and modified the surface property of substrate through oxygen plasma process. The others is changed the structure of substrate. It is hoped to reduce the usage of collagen and overcome hydrophobicity.

The specifications for the three substrates are as follows:

1. Flat substrate: flat PDMS substrate without any column structure and culture cells on it.

2. Large substrate: PDMS with an array of four columns (diameter $100 \mu \mathrm{m}$ ); culture cells on the cross flat area among the four columns; the width for flat area is $2 \mathrm{~mm}$.

3. Small substrate: PDMS with three columns (diameter $100 \mu \mathrm{m}$ ); column spacing is $100 \mu \mathrm{m}$; culture cells on the blank area between columns.

\section{Results and Discussion}

\subsection{Selection of Work Solution to avoid bubble formation}

The research used $\mathrm{NaCl}$ and glucose solution as the primary work solution. It was found in the beginning of cell culture, the adhesion was small, so it was easy for dielectrophoresis force to detach from the substrate. Once the cell adhesion became large, the applied voltage increases, but the tendency of electrolysis for aqueous solution also increased, and bubbles were formed on electrode surface and cell squeezing also occurred. The detachment of cells was not due to dielectrophoresis force. Formation of bubbles affected measurement of cell adhesion. Besides, formation of bubbles also tended to damage the electrodes.

Different solvent characteristic would have different concentration in water, so ion content and dissociation also vary, and there is different conductivity. The relationship between work voltage and solution to bubble formation is showed in Table 1. Voltage was applied at certain time intervals. For each concentration, five experiments were conducted and voltage at each bubble formation was recorded into table for comparison.

From the above results it was found that when glucose solution was used as work solution for adhesion force measurement high concentration solution was still easy to cause formation of bubbles on electrode surface, which affected measurement, while at low concentration measurement could be completed before formation of bubbles; with consideration that extremely low osmolarity would have impact to cell physiology or cell membrane rupture, it was suggested $2.0 \%$ glucose solution was the optimal work solution to assess human endothelial cell adhesion by dielectrophoresis force. 
Table 1. Work voltage of bubble formation under different work solution and concentration

\begin{tabular}{cccccccc}
\hline solution & Concentration $(\boldsymbol{\%})$ & $\mathbf{\# 1}$ & $\mathbf{\# 2}$ & $\mathbf{\# 3}$ & $\mathbf{\# 4}$ & $\mathbf{\# 5}$ & Voltage*(V) \\
\hline $\mathrm{NaCl}$ & 0.4 & 12.0 & 10.7 & 12.0 & 12.0 & 10.7 & 11.4 \\
$\mathrm{NaCl}$ & 0.9 & 8.2 & 8.2 & 8.2 & 8.2 & 8.2 & 8.2 \\
$\mathrm{NaCl}$ & 1.5 & 7.0 & 5.7 & 7.0 & 8.2 & 7.0 & 6.9 \\
$\mathrm{NaCl}$ & 2.0 & 10.7 & 10.7 & 10.7 & 9.4 & 10.7 & 10.4 \\
$\mathrm{NaCl}$ & 3.0 & 7.0 & 8.2 & 8.2 & 8.2 & 8.2 & 7.9 \\
glucose & 0.3 & 27.7 & 29.0 & 27.7 & 27.7 & 27.7 & 27.9 \\
glucose & 2.0 & 21.1 & 14.6 & 29.0 & 29.9 & 29.9 & 24.9 \\
glucose & 5.0 & 22.4 & 15.9 & 22.4 & 22.4 & 21.8 & 20.9 \\
glucose & 7.0 & 25.0 & 19.8 & 15.9 & 13.3 & 15.9 & 17.9 \\
glucose & 9.0 & 15.9 & 15.9 & 15.9 & 14.6 & 15.9 & 7.9 \\
\hline *(V): Average working
\end{tabular}

*(V): Average working voltage of bubble formation

\subsection{Style of cell culture substrate}

From Fig. 3 it was found that after surface modification by oxygen plasma contact angle changed a lot and the original hydrophobic nature was changed to hydrophilic. Therefore, after oxygen plasma treatment, experiment should proceed immediately to minimize errors due to surface property variation.

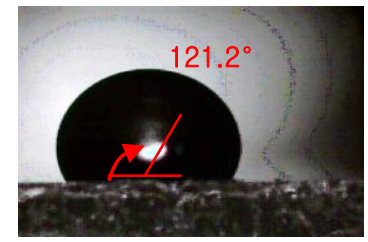

(a) Before treatment

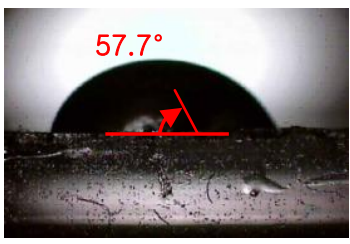

(b) after treatment

Fig. 3. Contact Angle before and after Oxygen Plasma Treatment

The experiment used $2.0 \%$ glucose solution as work solution with work frequency of $1 \mathrm{MHz}$, and used flat PDMS substrate treated by general method and oxygen plasma, and then cultured cells for $0 \sim 7$ hours prior conducting adhesion measurement.

Fig. 4 was adhesion diagram for flat substrate and indicated several parts that had abrupt drop of adhesion force. Since the culture dish and growth environment were identical and adhesion force was measured, theoretically adhesion force would increase with culture time and start leveling off after several hours. But the unusual phenomenon found in the curve suggested hydrophobic PDMS made it difficult for collagen evenly smeared, and under-smeared area could have significant decrease in adhesion force.

Fig. 4 was results of the adhesion curve measured with $2.0 \%$ glucose solution and oxygen plasma treated flat PDMS as cell culture substrate. It was found the maximum adhesion appeared after six hours and was about 15 $\mathrm{nN}$. The curve still showed several hours for abrupt drop of adhesion force. It was possibly because after PDMS substrate surface changed from hydrophobic to hydrophilic, although solution was easier to smear on the surface, the limited usage of collagen and no surface structure to hold the solution caused poor overall uniformity of smearing. 


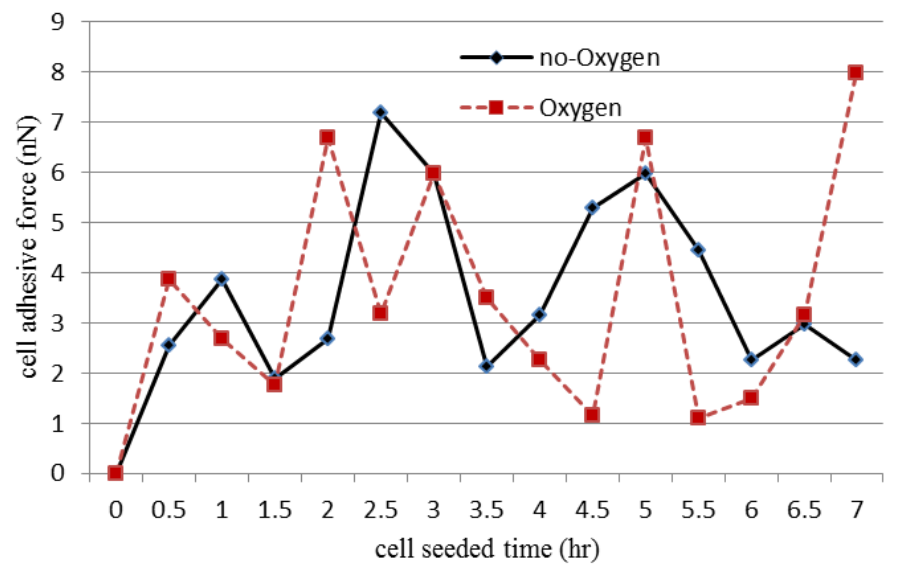

Fig. 4. Cell Culture Time and Cell Adhesion Force on Flat PDMS Substrate through oxygen plasma process

\subsection{Cell Adhesion Force for Different Microstructure}

To test collagen smearing state, besides the original flat substrate, there were another two different PDMS microstructures. There were flat substrate, large area substrate and small area substrate.

Fig. 5 showed the experimental results of cell adhesion curve with three different substrate microstructures when cells were cultured on large culture substrate and small culture substrate. Although both large culture substrate and small culture substrate showed few abrupt decreases in adhesion force, they were much less than flat substrate. When further comparison was made to the abrupt drop of adhesion force, it was also found that there was a trend that adhesion force increased with culture time.

From Fig. 5 it can be found that when collagen was smeared on microstructure, capillary force between columns would keep the solution in the gap. Therefore, besides adhesion curve could prove that structured PDMS had better adhesion; the pictures also indicated the existence of collagen between columns. When the cells were cultured on large area substrate or small area substrate, it was clear that their adhesion force increased with culture time.

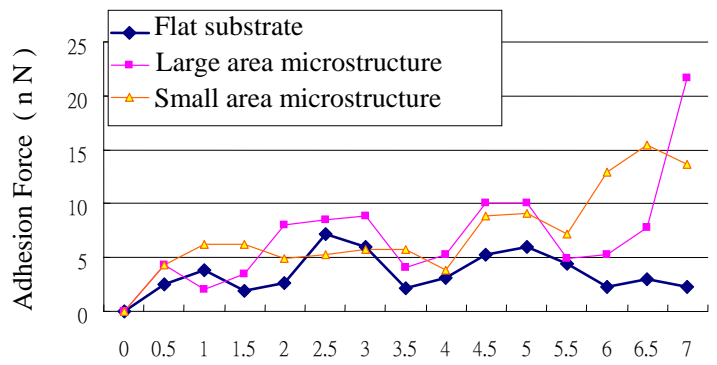

Cell seeded time (hr)

Fig. 5. Culture Time vs. Cell Adhesion Force for Substrate of Different Microstructures

From Fig. 5 it was found that among the three substrates the adhesion force was mostly lower in the flat substrate, and its maximum adhesion force occurred at 2.5 hours and the adhesive force was about $7.2 \mathrm{nN}$. 
Further, with increasing culture time, the adhesion force did not increase. When cells were cultured on large substrate and small substrate, the maximum adhesion force occurred at 7 and 6.5 hours and adhesion force was 21.6 and $15.4 \mathrm{nN}$. The large substrate had larger adhesion force than the smaller substrate.

\section{Conclusions}

The research is mainly to use dielectrophoresis force to measure cell adhesion force and establish the optimal measurement model. The conclusions are summarized in the following:

1. When $\mathrm{NaCl}$ is used as work solution and the concentration is around $0.4 \% \sim 3 \%$, it is easy to form bubbles and affect adhesion measurement. If weak electrolyte, $2.0 \%$ glucose solution, is used as work solution, adhesion measurement can be conducted smoothly.

2. When glucose solution is used as work solution, it is still possible to have bubble formation under voltage. Under such condition, it cannot make comparison for adhesion force under different osmolarity.

3. For oxygen plasma treatment on flat PDMS substrate, both experiment results and literatures indicated surface hydrophobicity was clearly improved. Compared to non-treated PDMS flat substrate, the treated one has better adhesion force.

4. When the endothelial cells are cultured on structured large PDMS substrate and small substrate, the maximum adhesion occurs at 7 and 6.5 hours and the forces are 21.6 and $15.4 \mathrm{nN}$, compared to $0 \mathrm{nN}$ before adhesion. The large substrate had larger average adhesion force than the small substrate. This means the large area substrate has better adhesion effect.

5. When the large area substrate with column spacing $2 \mathrm{~mm}$ is used to culture endothelial cells, it facilitates cell adhesion speed to obtain better growth condition. It is more suitable as the cell culture substrate for adhesion measurement.

6. Building structure on the substrate facilitates collagen smear uniformity. The structure also minimizes the usage of collagen and effectively assures that the cells to be measured are located in the smear area.

\section{References}

[1] O. Thoumine, A. Ott, D. Louvard, "Critical centrifugal forces induce adhesion rupture or structural reorganization in cultured cells." Cell Motil Cytoskeleton. Vol. 33, pp. 276-287, 1996.

[2] O. Thoumine, A. Ott, "Comparison of the mechanical properties of normal and transformed fibroblasts." Biorheology. Vol.34, pp.309-326, 1997.

[3] G. A. Truskey, T. L.Proulx, "Relationship between 3T3 cell spreading and the strength of adhesion on glass and silane surfaces." Biomaterials. Vol.14, pp.243-254, 1993.

[4] Z. Leonenko, E. Finot, M. Amrein, "Adhesive interaction measured between AFM probe and lung epithelial type II cells.” Ultramicroscopy, vol. 107, pp. 948-953, 2007.

[5] T. B. Jones, Electromechanics of particles. Cambridge, Cambridge University Press; 1995.

[6] R. Pethig, "Dielectrophoresis: using inhomogeneous AC electric fields separate and manipulate cells.” Crit. Rev. Biotechnology, vol.16, pp.331-348, 1996.

[7] H .Dalir, Y. Yanagida, T. Hatsuzawa, "Probing DNA mechanical characteristics by dielectrophoresis", Sensors and Actuators B. vol.136, pp.472-478, 2009.

[8] H. Morgan, M. P. Hughes, N. G. Green, "Separation of Submicron Bioparticles by Dielectrophoresis", Biophysical Journal.vol. 77, pp. 516-525, 1999.

[9] L. Benguigui, I. J. Lin, "More about the dielectrophoretic force", J. Appl Phys. Vol. 53, pp.1141-1143, 1982. 